\title{
Performance Evaluation of DWT-FDM and FFT-OFDM for Multicarrier Communications Systems using Time Domain Zero Forcing Equalization
}

\author{
R. Asif, R. A. Abd-Alhameed, O.O. Anoh and Y.A.S. Dama \\ School of Engineering, Design and Technology, \\ Bradford University, \\ Bradford, West Yorkshire, BD7 1DP, UK
}

\begin{abstract}
Other than the conventional fast Fourier transform (FFT) for multicarrier modulation, a new approach for multicarrier modulation (MCM) has been known. Meanwhile, multicarrier modulation involves dividing the broadband channel into many orthogonal but overlapping narrowband carriers. In an OFDM modulation based multicarrier system using the FFT, a cyclic prefix $(\mathrm{CP})$ is inserted after each symbol frame to combat the effects of inter symbol interference (ISI). By inserting the $\mathrm{CP}$, which results in spectral inefficiency OFDM schemes trades up to $25 \%$ of the transmit bandwidth. A new MCM approach that is void of the expense is the wavelet transform-based systems. These systems also have very suppressed side-lobes and exhibit improved BER performance. In wavelet based systems, the latest challenge in its implementation is in the channel estimation. In this work we have studied the performance of the FFT based OFDM system against wavelet transform (WT) based multicarrier system using a simple zero forcing (ZF) equalization in time domain. The studied system shows some improved BER performance.
\end{abstract}

\section{General Terms}

Equalization, Algorithm, Performance

\section{Keywords}

Orthogonal Frequency Division Multiplexing (OFDM), Bit error ratio (BER), Wavelet Transform (WT), Multicarrier Modulation (MCM)

\section{INTRODUCTION}

There is an ever-growing need for the communication systems that can provide high data rates. Modulation schemes characterized by theses high data rate transmissions can in turn incur ISI which is usually caused by the channel delay spread and as a result, high performance equalizers are required. Solution to this problem involves using multicarrier modulations (MCM), which divides the high data rate serial streams into a number of parallel streams with low data rates [1]. The number of sub-channels is selected so as to increase the symbol time as compared to the channel delay spread, and also to reduce the sub-stream bandwidth size than the size of the channel coherence bandwidth so the ISI can be bearable [2]. An instance of such MCM is the OFDM which is an FFTbased multicarrier modulation (MCM) scheme. OFDM modulation is the main contender for the communication systems required for the next generation. This method of multiplexing is rather expanded by the large side-lobes intrinsic in the fixed rectangular shaped FFT-window. This scheme also required a cyclic prefix insertion in order to compensate for the inter-symbol interference (ISI) which wastes up to $25 \%$ of the scarce bandwidth [3, 4]. Also, the OFDM signals are characterized by high peak-to-average power ratio (PAPR) which drives the high power amplifiers to operate near the saturation limits.

Wavelet Transform based multicarrier modulation is seriously being investigated as an alternative to the conventional Fast Fourier Transform for use in OFDM systems due to the fact that they can provide all the same benefits as of the FFTOFDM with an added benefit of PAPR combat and carrier frequency timing offset [5]. Unlike the conventional FFTOFDM system wavelet scheme satisfies the condition for orthogonality and achieves perfect reconstruction (PR) using the orthogonal filters of the Quadrature mirror filter (QMF) banks [6]. Discrete wavelet transform has large power spectral density as compared to FFT-OFDM due to the fact that DWT produce well contained side lobes with the narrower side lobe having the contained energy with reduced out-of-band emissions. Since there is no CP, the equivalent copied noise during cyclic prefixing is saved so that the BER performance of the WT-based systems is improved. Besides all these benefits wavelets also use multi-resolution analysis of the signal in which by the applied basis functions, the signal is well localized both in time and frequency domain and their resolution [7]. This multi-resolution- time-frequency signal representation is achieved by some shifting (translation) and scaling. Digital signal processing has played a very vital role in the advancement of the communication systems. By the filtering scheme in wavelets studies, the multirate filter banks have been in use in many fields such as digital communications, image processing and digital signal processing [8]. If we use the wavelet filters that allow the decomposition of the studied signals into equal high band and low band components of equal filter length, like the $\mathrm{db} 2$, and with the frequency components scaled in time, a simple zeroforcing $(\mathrm{ZF})$ can achieve a fair equalization wherein the channel state is known to the receiver.

The rest of this paper is divided as follows. Section II describes the implementation of the OFDM scheme, Section III covers wavelets as multicarrier modulation, simulation and results are presented in Section IV and Section V gives the conclusion.

\section{OFDM MODULATION}

\section{$2.1 \quad$ OFDM Transmitter}

Let us assume that we have an OFDM system that has $\mathrm{N}$ number of subcarriers. The subcarriers that will be used for transmission can be written as $\mathrm{Nu}+1$ which lie at the central 
spectrum and the subcarriers at the edges will form the guard bands. The transmission subcarriers are then modulated using a data symbol $X_{a, n}$ where ' $a$ ' is the number of the OFDM symbol and ' $\mathrm{n}$ ' stands for the subcarrier number. Inverse fast Fourier transform (IFFT) of size $\mathrm{N}$ is then applied. The subcarriers in the guard band are not utilized in order to keep the size of the transmit signal less than the bandwidth size of $1 / \mathrm{T}$ where $\mathrm{T}$ represents the sampling time of the OFDM signal. The addition of a guard band helps determine the best analog transmission filter to be employed in order to restrict the periodic spectrum at the output of the IFFT produced by the time signal (discrete). A guard interval also helps combat the inter symbol interference in a multipath fading channel environment. The resultant signal at the output of the transmitter can be mathematically written as [9]

$$
s(t)=\sum_{a=-\infty}^{\infty} \sum_{n=-N_{u} / 2}^{N_{u} / 2} X_{a, n} \Psi_{a, n}(t) \otimes g_{T}(t)
$$

Where, $\otimes$ represents the convolution, impulse response of the analog filter used in the transmission is given by $g_{T}(t)$ and $\Psi_{a, n}(t)$ stands for the subcarrier pulse and is defined as [9]

$$
\Psi_{a, n}(t)= \begin{cases}e^{j 2 \pi \frac{n}{T_{u}}\left(t-\Delta-a T_{s}\right),} & a T_{s} \leq t \leq(a+1) T_{s} \\ 0 & \text { otherwise }\end{cases}
$$

The total time of the OFDM symbol including the guard band will be equal to $T_{s}=T_{u}+\Delta$, OFDM subcarrier spacing will be equal to $1 / T_{u}$ and $\Delta$ is the length of the guard interval.

\subsection{Multipath Rayleigh Channel}

The signal was then transmitted over the Rayleigh fading channel which causes a change in phase, amplitude and the angle of arrival of the received signal and can be characterized by

$$
\mathrm{h}(\mathrm{t}, \tau)=\sum_{\mathrm{r}=1}^{\mathrm{R}} \mathrm{a}_{\mathrm{r}}(\mathrm{t}, \tau) \mathrm{e}^{-\mathrm{j} \theta_{\mathrm{r}}} \delta\left(\mathrm{t}-\tau_{\mathrm{r}}(\mathrm{t})\right)
$$

where, $\mathrm{a}_{\mathrm{r}}(\mathrm{t}, \tau)$, and $\tau_{\mathrm{r}}$ represent the amplitude and the time delay respectively and $\theta_{\mathrm{r}}=2 \pi \mathrm{f}_{\mathrm{c}} \tau_{\mathrm{i}}(\mathrm{t})$ is the phase shift for the $\mathrm{n}^{\text {th }}$ multipath at a time $\mathrm{t}$. $\mathrm{R}$ stands for the maximum number of multipath possible with $\delta(\bullet)$ as the Dirac delta. Thus, a Rayleigh fading channel is modeled as a complex-valued Gaussian process with zero mean.

\subsection{OFDM Receiver}

At the receiver it is assumed that the guard interval is longer than the channel delay spread, with perfect synchronization, and then the $\mathrm{n}^{\text {th }}$ subcarrier output during the $\mathrm{a}^{\text {th }}$ OFDM symbol can be written as [11]

$$
\begin{array}{r}
Y_{a, n}=X_{a, n} \cdot H_{a, n} \cdot G_{T}(n) G_{R}(n)+n_{a, n}, \\
-\frac{N_{u}}{2} \leq n \leq \frac{N_{u}}{2}
\end{array}
$$

Where, $n_{a, n}$ represents the additive white Gaussian noise, $G_{T}(n)$ stands for the frequency response of the analog transmission filter and $G_{R}(n)$ denotes the frequency response of the receiver filter at the nth subcarrier frequency $f_{n}=\frac{n}{T_{u}}$, channel response in the frequency domain is denoted as $H_{a, n}$ and can be mathematically explained as [11]

$$
H_{a, n}=\sum_{r=1}^{R} h_{n}\left(a T_{s}\right) \cdot e^{-j 2 \pi \frac{n T_{r}}{N T}}
$$

The channels $\mathrm{r}^{\text {th }}$ path gain during the $\mathrm{a}^{\text {th }}$ number of the OFDM symbol is represented by $h_{n}\left(a T_{s}\right)$ in Equation (5). Now if we assume that our transmission subcarriers lie in the flat region of the analog filters both at the transmitter and the receiver then we can rewrite the Equation (4) as [11]

$$
Y_{a, n}=X_{a, n} \cdot H_{a, n}+n_{a, n}
$$

Which mean we have assumed $G_{T}(n)$ and $G_{R}(n)$ to be equal to one in their flat region. Another way to eliminate $G_{T}(n)$ and $G_{R}(n)$ from Equation (4) will be to use the a priori knowledge of the transmitter and receiver filters. We have assumed Equation (6) to be correct for this study.

\section{MULTICARRIER MODULATION WITH WAVELETS}

\subsection{Wavelets Background}

Wavelet Transform (WT) is a mathematical function which can be used to decompose a continuous time signal at different scales and different times using the multi-resolution analysis [12].The continuous wavelet transform of a signal $\mathrm{x}(\mathrm{t})$ can be mathematically written as [12];

$$
\begin{aligned}
& \operatorname{CWTx}(\tau, a)=\left\langle x(t), \Psi_{\mathrm{a}, \tau}\right\rangle \\
& =\frac{1}{\sqrt{|\mathrm{a}|}} \int_{-\infty}^{\infty} x(\mathrm{t}) \psi^{*}\left(\frac{\mathrm{t}-\tau}{\mathrm{a}}\right) \mathrm{dt}
\end{aligned}
$$

Where ' $\psi$ ' represents the mother wavelet, parameter ' $\tau$ ' is the translation which corresponds to the time information present in the signal and ' $a$ ' is the scaling parameter which corresponds to the frequency information contained in the signal and ' $*$ ' represents the complex conjugate, which will not be required if ' $\psi$ ' is assumed to be real [13]

When a mother wavelet is designed it is designed in such a way that it can be inverted to retrieve the original transmitted signal. For continuous wavelet transform there exist no viable inverse in practice due to the redundancy in the information which will require extensive analytical calculation, but in theory (only mathematically) the inverse for this transform can be written as [6]:

$$
x(t)=\frac{1}{C_{\psi}} \int_{a} \int_{\tau} \frac{1}{a^{2}} x(a, \tau) \psi\left(\frac{t-\tau}{a}\right) d t d a
$$

Now to circumvent the above mentioned data redundancy issue we discretize the scaling and in addition the translation variables. Now using the Equation (7) as a reference if we discretize the translation parameter ' $\tau$ ' by $2^{\mathrm{j}} \mathrm{k}$, and scaling parameter 'a' by $2^{\mathrm{j}}$, then we can rewrite Equation (7) as:

$$
\begin{aligned}
\operatorname{DWTx}(j, k) & =\left\langle x, \psi_{j, k}\right\rangle \\
& =2^{\frac{-j}{2}} \sum_{n=-\infty}^{\infty} x(n) \psi\left(2^{-j} n-k\right)
\end{aligned}
$$

And thus the Equation (9) will be our discrete wavelet transform of signal $\mathrm{x}(\mathrm{t})$.This transform can also be referred to 
as a form of sub-band coding because in order to analyze a signal it has to pass through a string of filter banks [14].

\subsection{DWT scheme and Perfect Reconstruction}

The process involved in implementing the wavelet based MCM is quite similar to that of the conventional FFT-OFDM as follows;

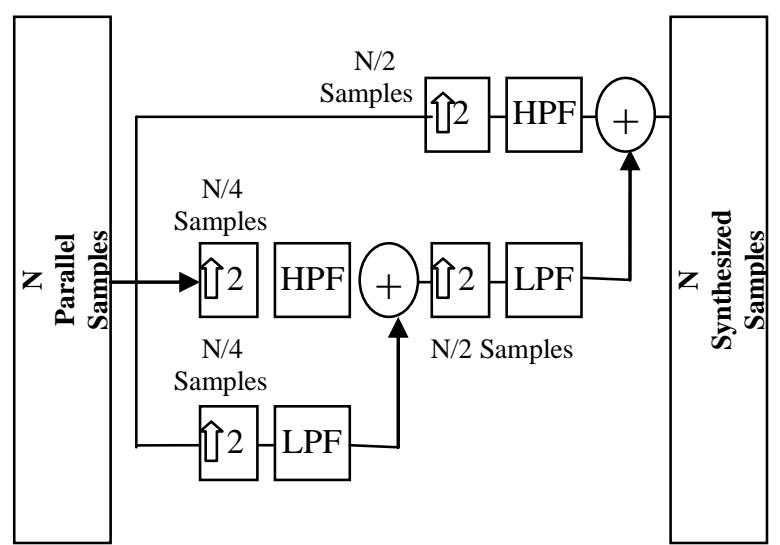

Figure 1: 2-Level Wavelet reconstruction (synthesis)

Wavelet transform based multicarrier modulated systems consist of perfect reconstruction quadrature mirror filter bank that employ half band low pass filter (LPF) whose impulse response can be written as ' $h$ ' and half band high pass filter (HPF) whose impulse response can be written as ' $\mathrm{g}$ ' which convolve with the input signal $x[n]$. Two sequential parallel data streams are created in wavelet transform multiplexing at each scale and translation i.e. $x_{\text {low }}(n)$ and $x_{\text {high }}(n)$ as shown in Figure (1) which are then upsampled by a factor of 2 and passed through the LPF and HPF of QMF bank. The input signal to each filter convolves with the impulse response of the filter to yield $x_{\text {low }}[n]=h[n] * x[n]$ for the low pass filter, which gives us our approximate coefficient and $x_{\text {high }}[n]=\mathrm{g}[n] * x[n]$ for the high pass filter which produces the detail coefficient at the filter output. Antiimaging filter is required in order to filter out the image frequencies that are produced during the upsampling operation within each channel. These filtered streams are then summed and constitute a wavelet symbol. This just explained process emphasizes synthesis (inverse DWT-IDWT) process. This synthesized data is then passed through the channel in the presence of AWGN.

When the signal is received on the receiver side it is then again passed through the Quadrature mirror filter bank which consists of a pair of conjugate LPF $h *(-n)$ and conjugate HPF g*(-n) on the receiver end. Two such pairs constitute a twochannel QMF bank as shown in Figure (2). The received signal is first decomposed into its respective detailed and approximate coefficients and then downsampled by a factor of 2 which is also apparent from the Figure (2). This process continues until the $\mathrm{N}$ parallel streams of data are recovered. The recovered data is then converted into a serial stream using a parallel to serial converter and then demodulated using a suitable scheme. In this study we have used BPSK and 16QAM.

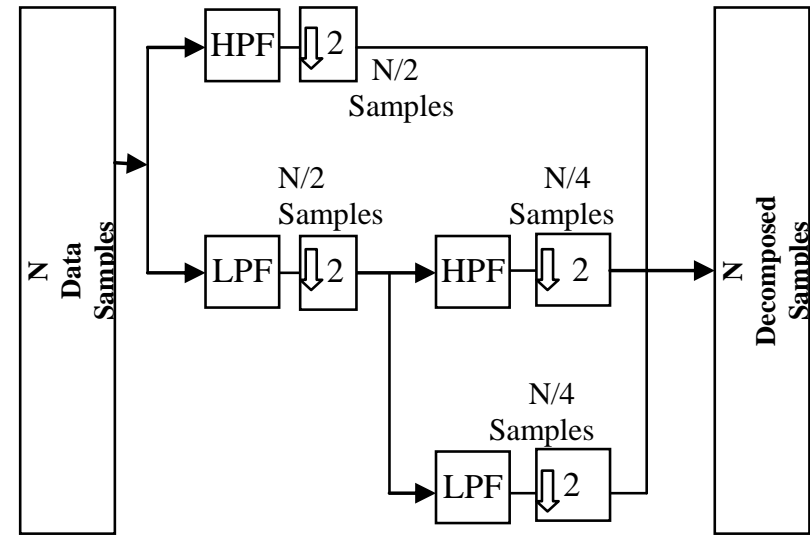

'Figure 2: 2-Level Wavelet Decomposition (Analysis)

The HPF and LPF of the QMF bank can be expressed mathematically as:

$$
h(n)=(-1)^{n} g(L+1-n)
$$

Where, $L$ describes the sequence of length of $g(n)$.

Because of the shift and translation of the wavelet transformed signals, each of the composite symbols is rather delayed by a factor ' $\alpha$ ' according to the $z$-transform relation $\left(\mathrm{X}(\mathrm{z})=\sum_{n} x(n) z^{-\alpha}\right.$, where $\left.z^{-\alpha}=e^{-j \alpha w}\right)$ which requires adjacent matched filters to perfectly reconstruct the signals. This perfect reconstruction can only hold if the matched filters respect the following.

$$
\begin{aligned}
& h(z) h^{*}(z)+g(z) g^{*}(z)=2 z^{-\alpha} \\
& h(z) h^{*}(-z)+g(z) g^{*}(-z)=0
\end{aligned}
$$

\section{Zero Forcing Equalization Algorithm}

One of the challenges faced when implementing a wavelet transform based multicarrier system is of the channel equalization. The use of zero-forcing channel equalization algorithm for wavelet transform based multicarrier systems using some finite response filters (FIR) was suggested in [15]. Meanwhile, $\mathrm{ZF}$ is adopted for channel equalization wherein the channel state is known to the receiver.

Table 1: Simulation Parameters for DWT and FFT in the presence of AWGN

\begin{tabular}{|c|c|c|}
\hline & DWT & FFT \\
\hline $\begin{array}{c}\text { Modulation } \\
\text { Scheme }\end{array}$ & $\begin{array}{c}\text { BPSK, 16- } \\
\text { QAM }\end{array}$ & $\begin{array}{c}\text { BPSK, and } \\
\text { 16-QAM }\end{array}$ \\
\hline FFT size & Nil & 64 \\
\hline Cyclic Prefix & Nil & $1 / 4$ \\
\hline DWT Family & Db2 & Nil \\
\hline $\begin{array}{c}\text { Decompositio } \\
\text { n Stages }\end{array}$ & $\mathrm{K}=\log 2(\mathrm{~N})$, & Nil \\
\hline Propagation & AWGN & AWGN \\
Environment & & \\
\hline Symbol & $2^{6} \times \mathrm{M}$, & $2^{6} \times \mathrm{M}$, \\
Length & $\mathrm{M}=10^{4}$ & $\mathrm{M}=10^{4}$ \\
\hline \multicolumn{2}{|c}{}
\end{tabular}


Without the ZF-with FIR approach, we can achieve a fair equalization in WT-transmissions since there are equal lengths of wavelet filters in both the receiver as of the transmitter for the wavelet family considered, db2. So, the zero forcing equalization algorithm is performed for WT and compared with that of the conventional FFT-OFDM over Rayleigh fading channel with some additive white Gaussian noise (AWGN).

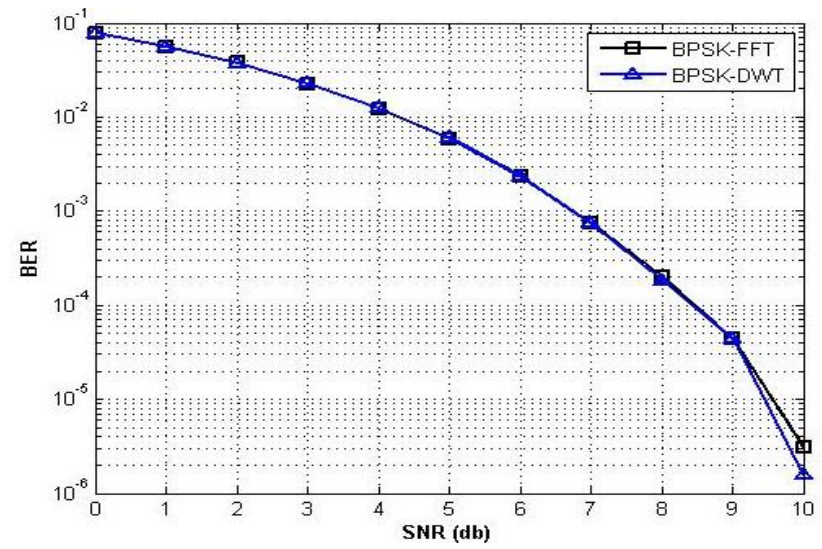

Figure 3: Performance comparison of DWT-OFDM and FFT-OFDM

The signal that reaches the receiver after the convolution with the channel and addition of noise can be mathematically written as $s_{\text {rec }}=h s+n$, where $h$ is the channel impulse response and $\mathrm{n}$ is the AWGN. In zero forcing equalization we need to find the filter coefficients $\mathrm{c}[\mathrm{t}]$ that can make $h[t] \otimes c[t]=\delta[t]$. By ZF, we proceed as follows;

$$
\begin{gathered}
\hat{y}_{Z F}[t]=c[t] \otimes y[t] \\
=c[t](s[t] \otimes h[t]+n) \\
=s[t]+c[t] \otimes n
\end{gathered}
$$

This $c[t] \otimes n$ causes noise amplification in zero forcing channel equalization. The equalized signal was then further processed for onward bit error ratio (BER) estimation.

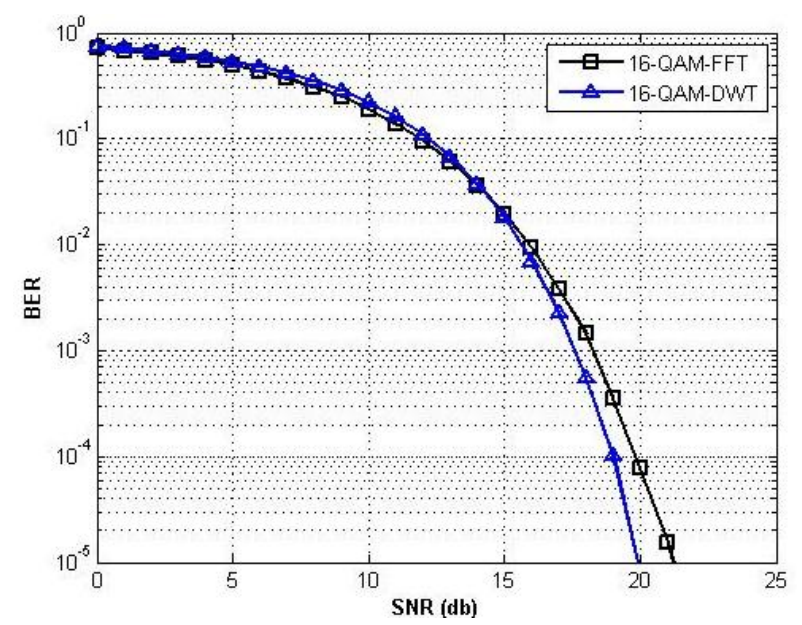

Figure 4: Performance comparison of DWT-OFDM and FFT-OFDM in AWGN using 16-QAM

\section{Simulation And Results}

In this section the results of the above mentioned system are discussed. Table 1 outlines the parameters that were used to carry out these simulations. The performance was studied using different modulation schemes in the presence of AWGN channel only as shown in Figures (3) and (4).

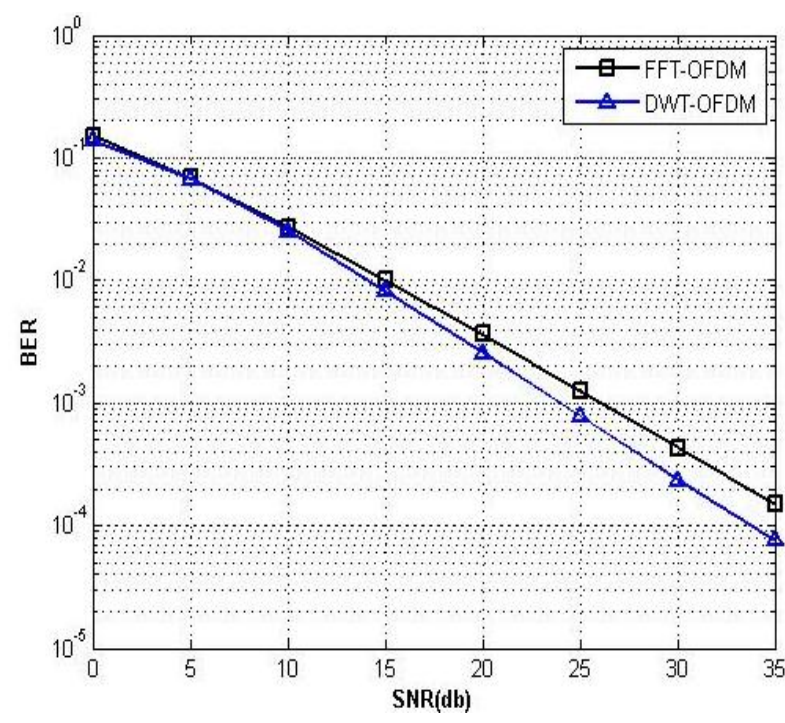

Figure 5: Performance comparison of DWT-OFDM and FFT-OFDM in Rayleigh Fading + AWGN using BPSK

It was noticed that the performance of the DWT was meritoriously marginal although with about $1 \mathrm{db}$ better at BER of $10^{-5}$ in the case of BPSK system in Figure (3) as compared to the conventional FFT-OFDM system. This performance can be better at some longer symbol length than the investigated. For higher data rate investigation, e.g. the 16QAM in Figure (4), the performance advantage of the DWTscheme becomes more pronounced with up to $2 \mathrm{~dB}$ gain at $10^{-4}$ BER in favor of the DWT modulation. The performance merit can be explained on the premise of cyclic prefixing required in the FFT-OFDM which is not required in the DWT-OFDM modulations since by copying 25 percent of each of the transmit symbol length, proportionate amount of noise is also copied. This would lower the likelihood of decoding the transmitted bits correctly.

Over the multipath, more disorientation are imposed on the transmit signal by the impulse response of the channel. But the signals processed by the DWT scheme posses some sturdy flexibility in time and frequency coupled the filtering mechanisms used in the wavelet. The filters of the wavelet used $(\mathrm{db} 2)$ decompose the signal into equal lengths of lowband and high-band and reconstructs them likewise. Since the channel state is known to the receiver, the effect of the channel can be removed with some bearable error introduced by the system noise. Comparing the performance of the DWTOFDM and FFT-OFDM in Figure (5), the results obtained from the simulation using discussed time domain equalization shows an improved BER performance and a gain of up to $3 \mathrm{db}$ at BER close to $10^{-4}$ in favor of DWT modulation when propagated through a multipath channel environment in the presence of AWGN using BPSK. 


\section{CONCLUSION}

In this study we implemented a wavelet transform based multicarrier system which comprised of perfect reconstruction Quadrature mirror filter banks and compared its performance against the conventional Fast Fourier transform based multicarrier system in the Rayleigh multipath fading environment with additive white Gaussian noise using zero forcing channel equalization algorithm which was implemented in time domain just before the DWT process. Results obtained showed slightly better BER performance in favor of the wavelet transform modulation. As no cyclic prefix or guard band was inserted spectral efficiency was improved in DWT as compared to the conventional FFTOFDM system where in order to counter the degrading effects of ISI cyclic prefixing is required. Consequently, further multicarrier modulation studies can be possible using the DWT-OFDM.

\section{REFERENCES}

[1] J. A. C. Bingham, "Multicarrier modulation for data transmission: An Idea Whose Time Has Come", IEEE Communications Magazine, vol. 28, no.5, pp. 5-14, 1990.

[2] Andrea Goldsmith, Wireless Communications, Cambridge University Press, (2005).

[3] G. Li and G.L. Stuber, Orthogonal frequency division multiplexing for wireless communications, Springer, 2006.

[4] Van De Beek, et al, Orthogonal frequency division multiplexing (OFDM), Review of Radio Science 199699, Intern. Union of Radio Science (URSI), 1999.

[5] V. Kumbasar and O. Kucur, "Better wavelet packet tree structures for PAPR reduction in WOFDM systems," Digital Signal Processing, 2008, Vol. 18, No. 6, pp. 885891.
[6] V. Kumbasar, et al., "Optimization of wavelet based OFDM for multipath powerline channel by genetic algorithm," Wireless Communications and Mobile Computing, 2009, Vol. 9, No. 9, pp. 1243-1250.

[7] Michael Weeks, Digital Signal Processing Using MATLAB and Wavelets, Georgia State University, 2007.

[8] Khan, U., Baig, S.: and Mughal, J. Performance Comparison of Wavelet Packet Modulation and OFDM for Multipath Wireless Channel with Narrowband Interference, 2nd International Conference on Computer, Control and Communication, pp1-4, (2009)

[9] Baoguo Yang; Letaief, K.B.; Cheng, R.S.; Zhigang Cao, Channel estimation for OFDM transmission in multipath fading channels based on parametric channel modeling, IEEE Transactions on Communications, Vol. 49, No. 3, 2001, pp. 467- 479.

[10] T.S. Rappaport, Wireless communications: principles and practice.Vol. 2, 1996: Prentice Hall PTR Upper Saddle River (New Jersey).

[11] Ye Li, G.L. Stuber, Orthogonal Frequency Division Multiplexing, Springer science + business media inc., 2006.

[12] N. Akansu and Richard A. Haddad, Multiresolution Signal Decomposition Transforms, Subbands, and Wavelets, Academic Press,(2001).

[13] I. Daubechies, Ten lectures on wavelets, Society for Industrial Mathematics, Vol. 61, 1992.

[14] M. K. Lakshmanan, and H. Nikookar, A Review of Wavelets for Digital Wireless Communication, Wireless Personal Communications, Vol.37, (2006), pp. 387-420.

[15] F. Farrukh, S. Baig, Mughal, M.J., "Performance comparison of DFT-OFDM and wavelet-OFDM with zero-Forcing equalizer For FIR Channel Equalization," International Conference on Electrical Engineering (ICEE),2007,pp.1-5. 